\title{
Prognostic Factors of Atypical Meningioma : Overall Survival Rate and Progression Free Survival Rate
}

\author{
Jae Ho Lee, M.D., ${ }^{1}$ Oh Lyong Kim, M.D., ${ }^{1}$ Young Beom Seo, M.D., Jun Hyuk Choi, M.D. ${ }^{2}$ \\ Departments of Neurosurgery, Pathology, Yeungnam University Medical Center, Yeungnam University College of Medicine, Daegu, Korea
}

Objective : Atypical meningioma is rare tumor and there is no accurate guide line for optimal treatment. This retrospective study analyzed the prognostic factors, the effect of different methods of treatments and the behavior of atypical meningioma.

Methods : Thirty six patients were diagnosed as atypical meningioma, among 273 patients who were given a diagnosis of meningioma in the period of 2002 to 2015. Age, gender, tumor location, Ki 67, Simpson grade and treatment received were analyzed. We studied the correlation between these factors with recurrence, overall survival rate and progression free survival.

Results : Median overall survival time and progression free survival time are 60 and 53 (months). Better survival rate was observed for patients less than 50 years old but with no statistical significance $(p=0.322)$. And patients with total resection compared with subtotal resection also showed better survival rate but no statistical significance $(p=0.744)$. Patients with a tumor located in skull base compared with patients with a tumor located in brain convexity and parasagittal showed better progression free survival $(p=0.048)$. Total resection is associated with longer progression-free survival than incomplete resection $(p=0.018)$.

Conclusion : We confirmed that Simpson grade was significant factor for statistically affect to progression free survival in univariate analysis. In case of skull base atypical tumor, it is analyzed that it has more recurrence than tumor located elsewhere. Overall survival was not affected statistically by patient age, gender, tumor location, Ki 67, Simpson grade and treatment received in this study.

Key Words : Malignant meningioma $\cdot$ Mortality $\cdot$ Progression free survival.

\section{INTRODUCTION}

Meningiomas are relatively slow-growing benign lesions arising from the arachnoid cells that form the meninges and account for $1326 \%$ of intracranial tumors. The World Health Organization (WHO) Classification of Tumors of the Central Nervous System recognizes three grades of meningio- mas. The choroid, clear cell, and common atypical meningiomas correspond to a WHO grade II classification. WHO grade III meningiomas are associated with aggressive growth patterns that reflect their clinical and histopathological features of malignancy and spread by metastatic dissemination. WHO grade I meningiomas most often occur in women and are associated with a relatively good outcome $e^{2,5,6,9,16)}$. Accord-

- Received : March 31, 2017 •Revised : June 29, 2017 • Accepted : July 3, 2017

- Address for reprints : Jae Ho Lee, M.D.

Department of Neurosurgery, Yeungnam University Medical Center, Yeungnam University College of Medicine, 170 Hyeonchung-ro, Nam-gu, Daegu 42415, Korea Tel : +82-53-620-3790, 3792, Fax : +82-53-620-3770, E-mail : sasimi9332@naver.com

This is an Open Access article distributed under the terms of the Creative Commons Attribution Non-Commercial License (http://creativecommons.org/licenses/by-nc/4.0) which permits unrestricted non-commercial use, distribution, and reproduction in any medium, provided the original work is properly cited. 
ing to the current WHO classification, between 5\% and 15\% of meningiomas are atypical (grade II), whereas 12\% are anaplastic meningiomas (grade III) ${ }^{13)}$. Because of the rarity and discordant pathologic criteria of atypical meningioma, the literature on the natural history and treatment of atypical meningioma is relatively scarce ${ }^{7,11}$. In comparison with the relatively good prognosis of benign meningiomas, atypical meningiomas are more locally aggressive and progress more rapidly. The distinction between benign and atypical or malignant meningioma represents important surgical information because surgical and treatment planning as well as prognostication will depend on the pathologic type ${ }^{20)}$. Gross total removal is the accepted standard of care for benign meningiomas; however, an optimal surgical management for atypical meningiomas has yet to be established ${ }^{10,12)}$. In addition, despite optimal surgery, local recurrences occur frequently. Putative predictive factors of tumor recurrence and overall survival (OS) in high-grade meningiomas are age, extent of resection, histological grade, and proliferative markers ${ }^{8,14,15,19)}$. This retrospective study analyzed the prognostic factors, effect of different treatment methods, and behavior of atypical meningioma.

\section{MATERIALS AND METHODS}

\section{Clinical material}

We retrospectively reviewed the records of all patients who were treated for atypical meningioma between 2002 and 2015 at Yeungnam University Medical Center. Among 273 patients who were diagnosed with meningioma, atypical meningioma was diagnosed in 41 patients. Subsequently, 36 patients were enrolled in this study; the other 5 patients were either lost to follow-up or had incomplete records and were excluded from this evaluation. Histology slides were not reviewed but all pathology reports were carefully examined. Data were collected from reviews of clinical records and neuroradiological investigations. Information on age, gender, tumor location, the presence or absence of postoperative radiotherapy, extent of surgical resection, and treatment received were individually obtained (Table 1). We studied the correlation between these factors and recurrence, OS, and progression-free survival (PFS). The extent of surgical resection was determined from operative notes or postoperative magnetic resonance imaging
(MRI) scans. Information on postoperative courses was obtained from records of outpatient clinics, phone contacts, and questionnaire by mail.

\section{Methods}

Patients were considered recurrent if there was a pathological documentation of recurrence, radiological documentation of recurrence or progression, or specific exacerbation of presenting symptoms. The indicated time to survival and absence of disease or recurrence/progression were calculated from the day of surgery. The median follow-up period was 63.9 months (range, 12158 months). Age at diagnosis was defined according to the date of first surgery for atypical meningioma. Surgical resection was evaluated according to the Simpson grading scale using the operative records and post-operative images. We defined total resection as Simpson grades I and II and incomplete resection or subtotal resection as Simpson grades III, IV or V. Complete resection (Simpson grade I or II) was achieved in 30 patients; the remaining 6 patients had incomplete resection (Simpson grade III, IV, or V). In addition, 6 patients received postoperative adjuvant conventional radiotherapy. Among them, 3 received whole brain radiotherapy $(60 \mathrm{~Gy}$, $50 \mathrm{~Gy}$, and $45 \mathrm{~Gy}$ ) and 3 received gamma knife radiosurgery.

Table 1. Clinical characteristics

\begin{tabular}{lc}
\hline Characteristic & Frequency \\
\hline Age & \\
$\quad<50$ & $9(25.0)$ \\
$\geq 50$ & $27(75.0)$ \\
Sex & \\
$\quad$ Male & $16(44.4)$ \\
Female & $20(55.6)$ \\
Location & \\
Skull base & $11(30.6)$ \\
Parasagittal & $12(33.3)$ \\
Convexity & $13(36.1)$ \\
Surgery & \\
Total (Simpson grade I-II) & $30(83.3)$ \\
Subtotal (Simpson grade III-V) & $6(16.7)$ \\
Ki-67 & \\
$>15 \%$ & $14(46.6)$ \\
$\leq 15 \%$ & $22(53.4)$ \\
RTx. & $6(16.7)$ \\
$(+)$ & $30(83.3)$ \\
$(-)$ &
\end{tabular}

Values are presented as number (\%). RTx. : radiation therapy 
Recurrence was defined as a radiological recurrence that corresponds to the radiological evidence of tumor regrowth in case of total resection, or to a residual tumor progressing, in case of incomplete resection. Recurrence was defined as 'radiological relapse', corresponding to radiological evidence of whether there was tumor progression in cases of incomplete resection (progression-free survival).

\section{Statistical analysis}

Statistical analysis was performed using SPSS software (Mi-

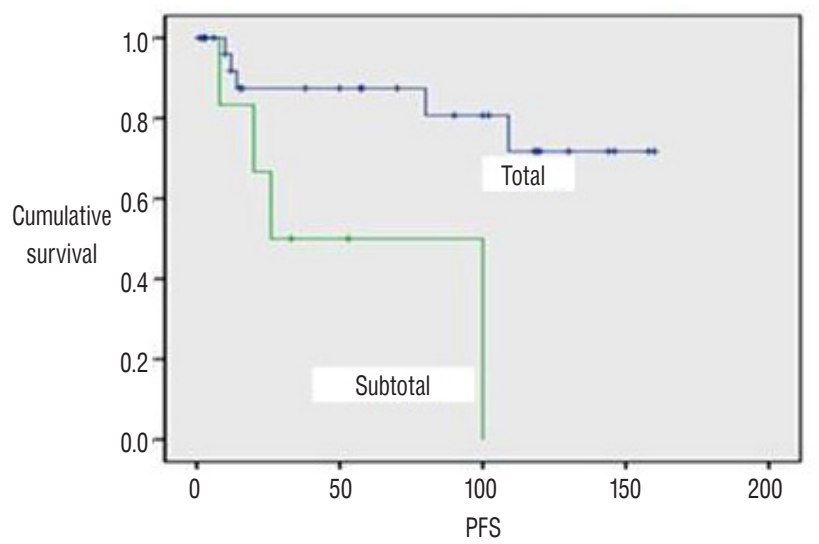

Fig. 1. The extent of surgical resection had a significant impact on PFS. PFS : progression free survival, Total : total removal of mass, Subtotal : subtotal removal of mass. crosoft Windows, Chicago, IL, USA). Survival statistics were based on time to death, measured from the age at diagnosis to the date of last follow-up or decease when related to meningioma surgery or progression. Survival function was assessed by the Kaplan-Meier method, and the Mantel-Cox log-rank test was used to compare different survival functions according to clinical and therapeutic factors. A $p$ value of $<0.05$ was considered as statistically significant. For the analysis, we considered radiotherapy as any form of radiation therapy.

\section{RESULTS}

Between 2002 and 2015, 273 meningiomas were operated on at our institute: of these, 232 (84.9\%) were benign, 41 (15.1\%) were atypical. Among 41 patients who diagnosed at atypical meningioma, 36 patients (5 patients were follow up loss) survived until the end of the follow-up period, and the 5 -year OS rate was $100 \%$. In our study, there was female predominance in atypical meningioma, and numbers of male and female were $16(44 \%)$ and 20 (56\%), respectively. The mean duration of preoperative symptoms and signs was 7 months. There were no differences between atypical and other meningiomas in relation to their typical symptoms and signs. The location of tumor were as follows : convexity of the skull $13(36.1 \%)$ patients; parasagittal 12 (33.3\%) patients; skull base $11(30.6 \%)$

Table 2. Statistical analysis of prognostic factors affecting PFS and OS

\begin{tabular}{|c|c|c|c|c|c|c|}
\hline \multirow{2}{*}{ Factor } & \multicolumn{4}{|c|}{ PFS } & \multicolumn{2}{|c|}{ OS } \\
\hline & Univariate & HR & Multivariate & HR & Univariate & HR \\
\hline \multicolumn{7}{|l|}{ Age } \\
\hline$\geq 50$ & 0.243 & 0.456 & ND & & 0.322 & 34.541 \\
\hline \multicolumn{7}{|l|}{ Sex } \\
\hline Female & 0.465 & 0.612 & ND & & 0.315 & 2.322 \\
\hline Skull base & 0.017 & 5.422 & 0.048 & 4.297 & 0.450 & 1.784 \\
\hline Parasagittal & 0.373 & 0.489 & ND & & 0.261 & 0.297 \\
\hline Convexity & 0.155 & 0.220 & ND & & 0.641 & 1.428 \\
\hline \multicolumn{7}{|l|}{ Surgery } \\
\hline Subtotal & 0.018 & 5.531 & 0.074 & 4.046 & 0.744 & 0.703 \\
\hline \multicolumn{7}{|l|}{ Ki-67 } \\
\hline$>15 \%$ & 0.316 & 1.966 & ND & & 0.377 & 1.966 \\
\hline \multicolumn{7}{|l|}{ RTx. } \\
\hline Yes & 0.296 & 2.115 & ND & 0.715 & 0.674 & \\
\hline
\end{tabular}

PFS : progression free survival, OS : overall survival, HR : hazard ratio, ND : not done, RTx. : radiation therapy 
patients. Total resection (Simpson grade I-II) was achieved for $30(80.3 \%)$ patients and subtotal resection (Simpson grade IIIV) for $6(16.7 \%)$ patients. There were no definite treatment guidelines; a small subgroup of patients underwent adjuvant radiotherapy treatment $(n=6)$. Mean patients age was $57.8 \pm$ 13.4 (27 to 80 years), and mean follow-up period was $63.9 \pm$ 55.4 (12 to 158 months). Median OS and PFS times were 60 (12 to 160 months) and 53 (8 to 160 months), respectively. Although the OS rate was better for patients less than 50 years old, it did not contribute significantly to PFS ( $p=0.096$ ). The extent of surgical resection had a significant impact on PFS; PFS was $65.1 \pm 55.1$ in patients with gross total resection compared with $40.0 \pm 32.9$ in patients with subtotal resection ( $p=0.008$ ) (Fig. 1). The survival rate of patients with total resection (Simpson grade I-II) was also better compared with that of patients with subtotal resection (Simpson grade III-V); however, there was no statistical significance $(p=0.744)$. Patients with skull base tumors had more recurrence rate and there was statistical significance $(p=0.048)$. No relationship was between Ki-67 labeling index and OS or PFS was observed ( $p=0.377, p=0.316$ ). Although not statistically significant, OS was longer in younger patients (Table 2). All specimens were reviewed by one neuropathologist and confirmed as atypical meningioma.

\section{DISCUSSION}

Atypical meningioma is rare tumor and there is no accurate guideline for optimal treatment. Despite its methodological limitations, this study is the largest series in the literature on the outcome and prognostic factors that affect the survival rates of atypical meningioma. Of all meningioma subtypes, atypical meningioma represents 4 to $7 \%{ }^{10,14)}$. This was a retrospective study; thus, one of the limitations of this study could be random bias. In addition, we could not provide the same level of evidence as a prospective study or randomize control trial. Since these were the results of a single center and single surgeon, there could also be a selection bias. Furthermore, there were insufficient cases to compare for different variables because of a rare atypical meningioma. The future direction of this prospective study is expected to be challenging in identifying several prognostic factors of atypical meningioma, as patients with atypical meningioma are rare. Nevertheless, in comparison with previous studies in Korea, our study focused on recent patients in the past 10 year. Therefore, it would have the advantage of utilizing more advanced imaging equipment and surgical equipment. Consequently, surgical removal can be considered easier, and information such as tumor proliferation index (Ki-67) levels and brain invasion on MRI can potentially be new prognostic factors. These would be more efficient in reflecting the latest trends of atypical meningioma

After a review of previous studies prognostic factors associated with OS, PFS are the age at the time diagnosis, surgical removal, tumor proliferation index, tumor location, and adjuvant radiotherapy. We performed a comparison of these factors in our study.

\section{Age}

There was no statistical significance of recurrence when compared with age and different factors at the same time. However, age at diagnosis was significantly meaningful as prognostic factors for OS and PFS were lower than the average. Champeaux et al. ${ }^{5)}$ reported that those younger than 57 years had fewer operations than those above 57 years old since recurrence and OS were shown to be associated with age at diagnosis. Aghi et al. ${ }^{1)}$ found that age at diagnosis has already been reported to be associated with the overall survival of atypical meningioma.

\section{Extent of resection}

According to the Zaher et al. ${ }^{22)}$ there is a general agreement about the importance of resection completeness, and it is clear that subtotally removed meningiomas may continue to grow. The extent of resection (Simpson grading) is the most powerful prognostic factor of recurrence for all grades of meningiomas. Total resection is associated with better local control than incomplete resection. Cao et al. ${ }^{4)}$ recommended that total resection of the tumors under relatively safe conditions remains the suitable strategy of treatment.

In this study, we investigated the statistical significance of PFS and OS with various factors. The results suggest that the PFS of total removal was increased compared with that of subtotal removal. Although the pathological type of tumor is very important for prognosis but the extent of tumor resection is also very important factor. 


\section{Tumor proliferation index}

The Ki-67 protein is a cellular marker for proliferation. There is a strong correlation in meningioma between histology and Ki-67 antigen expression, which can be detected using the Ki-67 monoclonal anti body ${ }^{3}$. However, the relationship between proliferation and clinical outcome is still controversial. Certain studies indicate that there is no correlation between clinical outcomes and proliferation ${ }^{17,21)}$. Nevertheless, Park et al. ${ }^{18)}$ confirmed that Ki-67 levels greater than $15 \%$ are a useful predictor of recurrence. And Bruna et al. ${ }^{3)}$ demonstrated that Ki-67 labeling index level is the only independent predictor of both tumor recurrence and overall survival. However, experience as well as the literature indicates interlaboratory variability in staining reproducibility and labeling index assessment.

\section{Tumor location}

Hug et al. ${ }^{11)}$ found that the majority of atypical meningiomas occurred in the cerebral convexity. In this paper, in the comparison between convexity atypical meningioma and skull base, recurrence rate was increased in skull base meningioma. However, when comparing between convexity lesion and skull base, we could not determine which is more biologically malignant. Skull base lesions are more difficult for total resection than convexity lesion, as shown in these results. Further studies would be necessary in the future.

\section{CONCLUSION}

Atypical meningioma is rarely reported intracranial tumor. In our study, atypical meningioma shows aggressive nature and we confirmed that Simpson grade and tumor location were a significant factor that statistically affected PFS. This was similar to the results of a previous study. With long-term follow up period, it would be able to observe more independent prognostic factors clearly. We conclude that surgical complete resection may have a significant impact on PFS and may be helpful in determining the direction of treatment for future atypical meningioma patients.

\section{References}

1. Aghi MK, Carter BS, Cosgrove GR, Ojemann RG, Amin-Hanjani S, Martuza $\mathrm{RL}$, et al. : Long-term recurrence rates of atypical meningiomas after gross total resection with or without postoperative adjuvant radiation. Neurosurgery 64 : 56-60; discussion 60, 2009

2. Akeyson EW, McCutcheon IE : Management of benign and aggressive intracranial meningiomas. Oncology (Williston Park) 10 : 747-756; discussion 756-759, 1996

3. Bruna J, Brell M, Ferrer I, Gimenez-Bonafe P, Tortosa A : Ki-67 proliferative index predicts clinical outcome in patients with atypical or anaplastic meningioma. Neuropathology 27 : 114-120, 2007

4. Cao X, Hao S, Wu Z, Wang L, Jia G, Zhang L, et al. : Treatment response and prognosis after recurrence of atypical meningiomas. World Neurosurg 84 : 1014-1019, 2015

5. Champeaux C, Wilson E, Brandner S, Shieff C, Thorne L : World Health Organization grade III meningiomas. A retrospective study for outcome and prognostic factors assessment. Br J Neurosurg 29 : 693-698, 2015

6. Chen WY, Liu HC : Atypical (anaplastic) meningioma: relationship between histologic features and recurrence--a clinicopathologic study. Clin Neuropathol 9 : 74-81, 1990

7. Coke CC, Corn BW, Werner-Wasik M, Xie Y, Curran WJ Jr : Atypical and malignant meningiomas: an outcome report of seventeen cases. J Neurooncol 39 : 65-70, 1998

8. Condra KS, Buatti JM, Mendenhall WM, Friedman WA, Marcus RB Jr, Rhoton AL : Benign meningiomas: primary treatment selection affects survival. Int J Radiat Oncol Biol Phys 39 : 427-436, 1997

9. Dziuk TW, Woo S, Butler EB, Thornby J, Grossman R, Dennis WS, et al. : Malignant meningioma: an indication for initial aggressive surgery and adjuvant radiotherapy. J Neurooncol 37 : 177-188, 1998

10. Goyal LK, Suh JH, Mohan DS, Prayson RA, Lee J, Barnett GH : Local control and overall survival in atypical meningioma: a retrospective study. Int J Radiat Oncol Biol Phys 46 : 57-61, 2000

11. Hug EB, Devries A, Thornton AF, Munzenride JE, Pardo FS, HedleyWhyte ET, et al. : Management of atypical and malignant meningiomas: role of high-dose, 3D-conformal radiation therapy. J Neurooncol 48 : 151-160, 2000

12. Jo K, Park HJ, Nam DH, Lee Jl, Kong DS, Park K, et al. : Treatment of atypical meningioma. J Clin Neurosci 17 : 1362-1366, 2010

13. Louis D, Scheithauer B, Budka H, Von Deimling A, Kepes J, Kleihues P, et al. : Pathology and genetics of tumors of the nervous system in Kleihues P, Cavenee WK (eds) : World Health Organization Classification of Tumours: Pathology and Genetics: Tumors of the Nervous System, ed 2. Lyon : IARC Press, 2000, Vol 1, pp176-184

14. Mahmood A, Caccamo DV, Tomecek FJ, Malik GM : Atypical and malignant meningiomas: a clinicopathological review. Neurosurgery 33 : 955-963, 1993

15. McCarthy BJ, Davis FG, Freels S, Surawicz TS, Damek DM, Grutsch J, et al. : Factors associated with survival in patients with meningioma. J Neurosurg $88: 831-839,1998$ 
16. Modha A, Gutin PH : Diagnosis and treatment of atypical and anaplastic meningiomas: a review. Neurosurgery 57 : 538-550; discussion 538550, 2005

17. Palma L, Celli P, Franco C, Cervoni L, Cantore G : Long-term prognosis for atypical and malignant meningiomas: a study of 71 surgical cases. J Neurosurg $86: 793-800,1997$

18. Park HJ, Kang HC, Kim IH, Park SH, Kim DG, Park CK, et al. : The role of adjuvant radiotherapy in atypical meningioma. J Neurooncol 115 : 241-247, 2013

19. Perry A, Stafford SL, Scheithauer BW, Suman VJ, Lohse CM : Meningioma grading: an analysis of histologic parameters. Am J Surg Pathol
$21: 1455-1465,1997$

20. Tomura N, Takahashi S, Sakuma I, Omachi K, Watarai J, Sasajima T, et al. : Neuroradiological findings of atypical meningiomas. CMIG Extra: Cases $28: 33-39,2004$

21. Torp SH, Lindboe CF, Granli US, Moen TM, Nordtømme T : Comparative investigation of proliferation markers and their prognostic relevance in human meningiomas. Clin Neuropathol 20 : 190-195, 2001

22. Zaher A, Abdelbari Mattar M, Zayed DH, Ellatif RA, Ashamallah SA : Atypical meningioma: a study of prognostic factors. World Neurosurg $80: 549-553,2013$ 